\title{
General
}

\section{Attachment Styles, Personality, and Frustration Intolerance}

\author{
Christian Schetsche ${ }^{1}$ (D) Alba E. Mustaca² (D) \\ 1 Instituto de Investigaciones, Facultad de Psicología, Universidad de Buenos Aires, ${ }^{2}$ Facultad de Psicología y Relaciones Humanas, Universidad \\ Abierta Interamericana \\ Keywords: structural equation modeling, frustration intolerance, personality traits, attachment styles \\ https://doi.org/10.52965/001c.24551
}

\section{Health Psychology Research}

Vol. 9, Issue 1, 2021

The objective of the present study was to evaluate the relationships between attachment styles, personality traits, and their effects on frustration intolerance (FI) in a sample of 300 adults. The Argentine Attachment Styles Scale, the Eysenck Revised Personality Questionnaire (EPQ-RA), and the Frustration Intolerance Scale (EIF) was used. In the two Full Latent Variable Models developed, it was determined that neuroticism and avoidant attachment had the greatest mediating effects on FI. Avoidant attachment stood out for its mediating effect on anxious attachment. These two factors were found to explain a considerable percentage of neuroticism and, as a consequence, the four dimensions of FI. It is concluded that it is necessary to reinterpret certain concepts of neuroticism and study them from a perspective that starts from attachment theory.

\section{INTRODUCTION}

Amsel defines frustration as the organism's state triggered by the unexpected devaluation, omission, or inaccessibility of a positive reinforcer. ${ }^{1}$ Frustration causes physiological, behavioral, and neural responses analogous to the presentation of aversive stimuli; they are similar to sensory pain, fear, and anxiety. ${ }^{2}$ Ellis, in the clinical area, considered that psychological maladjustments originate in part from a high intolerance to frustration caused by irrational beliefs, in line with basic studies that show a more significant frustration when there is a high discrepancy between what is expected and what is obtained. ${ }^{3}$

FI has been related to personality; Eysenck's psychobiological theory is one of the main ones for having developed studies on the neurological bases for this relationship. ${ }^{4}$ Eysenck developed a hierarchical model that ranges from the most general dimensions (extraversion, neuroticism, and psychoticism) to the most particular (specific traits, habits, and behaviors). ${ }^{5}$ The dimensions were regarded as independent of each other, but current research has shown that they overlap conceptually, as neuroticism correlates negatively with extraversion and positively with psychoticism. ${ }^{6,7}$ High neuroticism scores predict many mental disorders, such as comorbidity, ${ }^{8-11}$ and negative coping styles. ${ }^{12}$ In addition, they are associated with a variety of physical problems: Cardiovascular disease, eczema, asthma, and irritable bowel syndrome. ${ }^{13,14}$ These relationships remain significant in longitudinal investigations. ${ }^{15}$ Regarding FI, direct correlations were found with high neuroticism. ${ }^{16-18}$

Genetic load and early and environmental experiences, in general, are interrelated in a complex way with the etiology of individual differences in all behavior. Studies with animal and human models found that early experiences have a significant impact on the development of individual differences, generating a wide range of responses to stressful situations in adulthood, from greater vulnerability to greater resilience. ${ }^{2,19-25}$

Personality is 40 to $50 \%$ determined by genetic factors. ${ }^{26}$ Regarding the environmental factors that influence personality and attachment styles were among the most studied. Bowlby's attachment theory emphasizes that the child's early experiences with his/her caregivers are crucial for optimal development; ${ }^{27}$ the type of bond established between them depends fundamentally on the sensitivity and responsiveness of the adult concerning the needs of the child. ${ }^{28}$ Through the strange situation experiment, ${ }^{29}$ three classifications of attachment styles in children, emerged: secure, insecure-avoidant, and insecure-ambivalent. The proportion of children with secure attachment correspond to $66 \%$ of the total; $22 \%$ with insecure-avoidant attachment, and $12 \%$ with insecure-ambivalent attachment. ${ }^{29,30}$

Numerous studies found that anxious and avoidant attachment have positive correlations with neuroticism and negative associations with extraversion. ${ }^{31}$ In another study, Jenkins-Guarnieri, and colleagues found associations between anxious attachment and these two personality traits. ${ }^{32}$ Barel and colleagues evaluated the relationships between attachment style, personality, and temperament in adults. ${ }^{33}$ They found that socio-environmental factors (perceived attachment styles in adults) are important moderating elements by linking personality variables with temperament. Furthermore, Mikulincer and Shaver describe the mechanism through which the unavailability of the attachment figure can lead to FI, ${ }^{34}$ and Norberg and colleagues found associations between high FI and anxious attachment. 35

To analyze the relationships between personality and attachment styles and to determine the most significant predictors of FI, in this research, two Full Latent Variable Models were developed: one that maximizes the explained 
variances and the other that, interpreting secure attachment as a protective element, ${ }^{36}$ used this factor as an independent variable.

The Ethics Committee approved this research of the Universidad Abierta Interamericana, Buenos Aires, Argentina.

\section{METHOD}

\section{PARTICIPANTS}

Intentional sampling was conducted, and the sample was made up of 300 adults ( $\mathrm{M}$ age $=31.98, \mathrm{SD}=11.93$, female $=205$ ) residing in Argentina. 68\% had an incomplete or higher university level. Taking into account the high number of female participants, it was decided to perform a multigroup analysis according to gender because it allows determining the factorial invariance of the model. ${ }^{37}$

\section{INSTRUMENTS}

Argentine Scale of Attachment Styles (EAEA). ${ }^{38}$ Through a four-point Likert scale $(1=$ Almost never to $4=$ Almost always), 20 items are answered, which assess the perception of secure, anxious, and avoidant attachment in romantic and non-romantic ties. The original validation study obtained Cronbach's alphas of $0.45 \leqslant \alpha \leqslant 0.52$.

Eysenck's Revised Personality Questionnaire (EPQ-RA), Argentine version by Squillace and colleagues. ${ }^{39}$ It has 42 items that present the dimensions of psychoticism, extraversion, neuroticism, and sincerity; it is answered on a dichotomous scale (Yes-No). The authors obtained Cronbach's alphas $0.66 \leqslant \alpha \leqslant 0.84$. Since the sincerity factor is used to estimate the reliability with which the participant responds to the questions, it was excluded in the present study, having previously confirmed that they had acceptable levels.

Frustration Intolerance Scale (EIF), the Argentine version of Medrano and colleagues. ${ }^{40}$ Irrational beliefs that promote FI are evaluated with 17 items on a 5-point Likert scale $(1=$ It is not at all characteristic of me to $5=$ It is very characteristic of me). High scores demonstrate higher FI. These items are grouped into four dimensions: intolerance of discomfort, demand for affection and rights, emotional intolerance, and intolerance to the absence of achievement. In the original validation study, the subscales presented Cronbach's alphas of $0.61 \leqslant \alpha \leqslant 0.84$.

Ad hoc sociodemographic questionnaire. This inquired about age, gender, educational level, if the participant was undergoing any psychological treatment, and if the participant currently has a stable partner.

\section{DATA ANALYSIS}

The Local Outlier Factor (LOF) test was performed based on density analysis to detect multivariate outliers. ${ }^{41}$ For this, we used the dbscan package. ${ }^{42}$ The calculation of multivariate normality was carried out with the $M V N,{ }^{43}$ and the Structural Equation Modeling (SEM), its development and adjustment, the calculation of the effects, and the factorial invariance analysis were carried out with the lavaan package. ${ }^{44}$ Only latent variables (Full Latent Variable Model) were used. All the mentioned extensions are part of the $\mathrm{R}$ software $;{ }^{45}$ A probability value $=p \leqslant 0.05$ was used as a significance criterion.

\section{RESULTS}

\section{OUTLIERS AND MULTIVARIATE NORMALITY}

We located 45 severe multivariate outliers, so they were excluded from the sample, reducing it to $n=255$. Using Mardia's test, ${ }^{46}$ we found the non-existence of multicollinearity and that the initial 79 items did not represent multivariate normality. Due to this and the fact that the EPQ-RA has a dichotomous scale, we used the weighted least squares method with robust standard error (WLSMV) to evaluate model fits. ${ }^{47-49}$

According to $\mathrm{Hu}$ and Bentler's suggestions, ${ }^{50}$ a model is considered adequate when its fit indices are: SRMR (standardized root mean square residual) $\leqslant 0.08$, RMSEA (root mean square error of approximation) $\leqslant 0.06$, CFI (comparative fit index) $\geqslant 0.95$, and TLI (Tucker Lewis Index) $\geqslant 0.95$. Following preacher and Hayes' indications, ${ }^{51}$ we used the bootstrapping method since it calculates confidence intervals for the regression analysis. This is especially important when calculating indirect effects and/or when data do not comply with the assumption of multivariate normality. Taking into account the sample size, the percentile-based method was used with 500 bootstrap samples. ${ }^{52-54}$

\section{PURIFICATION MEASURES}

In SEM, residuals are important, so Hair and colleagues recommend carefully analyzing items that exhibit many standardized residuals (> 2.50) with other items. ${ }^{55}$ Applying this criterion, we carried out a purification measure and reduced all the instruments to three items per dimension. After this, the following items represented their respective factors: neuroticism, 12, 18, 36; extraversion, 6, 11, 24; psychoticism, 16, 22, 39; anxious attachment, 2, 8, 13; avoidant attachment, 3, 6, 16; secure attachment, 7, 11, 17; emotional intolerance, 2, 7, 11; demand for affection and rights, 10, 13, 15; intolerance of discomfort, 3, 9, 12; and intolerance to absence of achievement, 8, 14, 17.

As shown in Table 1, the psychometric properties improved substantially, thus complying with all the fit indices proposed by $\mathrm{Hu}$ and Bentler. ${ }^{50}$ Thus, we used the brief versions EPQ-RA (9), EAEA (09), and EIF (12) for the development of the Full Latent Variable Models.

\section{DEVELOPMENT OF THE FIRST FULL LATENT VARIABLE MODEL}

\section{DIRECT EFFECTS OF PERSONALITY TRAITS AND ATTACHMENT STYLES ON FI}

According to the first objective, we used the four dimensions of FI as dependent variables and personality traits and attachment styles as independent variables. As evidenced in Table 2, only neuroticism, anxious attachment, and psychoticism directly affected the FI scales. 
Table 1. Fit indices of confirmatory factor analysis

\begin{tabular}{|c|c|c|c|c|c|c|c|c|c|}
\hline $\begin{array}{l}\text { Competing } \\
\text { models }\end{array}$ & $\begin{array}{c}\text { X2 } \\
\text { WLSMV }\end{array}$ & $d f$ & $x 2 / d f$ & Scaling & $\mathrm{p}$ & $\begin{array}{c}\text { RMSEA } \\
(90 \% \mathrm{Cl})\end{array}$ & SRMR & CFI & TLI \\
\hline $\begin{array}{l}\text { EPQ-RA } \\
\text { (42) }\end{array}$ & 1159,701 & 813 & 1,426 & 2,007 & 0,000 & $\begin{array}{c}0,041(0,036 \\
-0,046)\end{array}$ & 0,078 & 0,781 & 0,768 \\
\hline EPQ-RA (9) & 52,135 & 48 & 1,086 & 0,929 & 0,316 & $\begin{array}{c}0,018(0,000 \\
-0,046)\end{array}$ & 0,045 & 0,985 & 0,979 \\
\hline EAEA (20) & 245,738 & 167 & 1,471 & 0,813 & 0,000 & $\begin{array}{c}0,043(0,031 \\
-0,054)\end{array}$ & 0,062 & 0,893 & 0,878 \\
\hline EAEA (09) & 26,133 & 24 & 1,089 & 0,538 & 0,346 & $\begin{array}{c}0,019(0,000 \\
-0,056)\end{array}$ & 0,040 & 0,991 & 0,987 \\
\hline EIF (17) & 239,255 & 113 & 2,117 & 0,766 & 0,000 & $\begin{array}{c}0,066(0,055 \\
-0,078)\end{array}$ & 0,065 & 0,907 & 0,888 \\
\hline EIF (12) & 78,796 & 48 & 1,642 & 0,516 & 0,003 & $\begin{array}{c}0,050(0,029 \\
-0,070)\end{array}$ & 0,045 & 0,966 & 0,953 \\
\hline
\end{tabular}

Notes. $\mathrm{n}=255 ; \chi 2$ WLSMV - Chi-square using weighted least squares method with robust standard error; df, degrees of freedom; Scaling, scaling correction factor for de WLSMV estimator; RMSEA, root mean square error of approximation; SRMR, standardized root mean square residual, CFI, comparative fit index; TLI, Tucker Lewis Index; number in parentheses, number of items.

\section{DIRECT EFFECTS BETWEEN PERSONALITY TRAITS AND ATTACHMENT STYLES}

Table 3 shows all the direct effects between personality traits and attachment styles. We found several reciprocal effects: Anxious attachment with extraversion, avoidant attachment with psychoticism, anxious with avoidant attachment, and avoidant with secure attachment.

\section{ESTABLISHMENT OF THE FIRST FULL LATENT VARIABLE $M O D E L$}

In developing SEM, reciprocal paths can emerge. Although these may be legitimate, Byrne states that not all of them may be of interest. ${ }^{37}$ That is why in the first model, the four dimensions of FI were used as dependent variables and, to establish the relationships between personality traits and attachment styles, we continued working with the direct effect with a lower probability value $p$. In this way, we found the first SEM. Still, we noted that the effect of avoidant attachment on psychoticism and the effects of anxious attachment on intolerance to discomfort and emotional intolerance were no longer significant, so we excluded them from the model. Using the method proposed by Saris and colleagues, ${ }^{56}$ we reviewed the modification indices and added a direct effect of anxious attachment on neuroticism.

As reflected in Table 4, the following effects are highlighted as the most significant of the first model: avoidant attachment on secure attachment, anxious attachment on neuroticism, neuroticism on intolerance to discomfort, and neuroticism on demand for affection and rights.

Fit indices were calculated and the first model obtained the following values: $\chi 2$ WLSMV (Chi-square using the weighted least squares method with robust standard error) $=421.268 ; \mathrm{df}=380 ;$ Scaling (standard error correction adjustment factor $)=1.290 ; \mathrm{p}=0.085 ;$ RMSEA $=0.021,90 \% \mathrm{CI}$ $[0.000 ; 0.031]$; SRMR $=0.051 ; \mathrm{CFI}=0.971$ and $\mathrm{TLI}=0.966$. According to the suggestions of $\mathrm{Hu}$ and Bentler, 50 it can be stated that a significantly accurate model was developed.

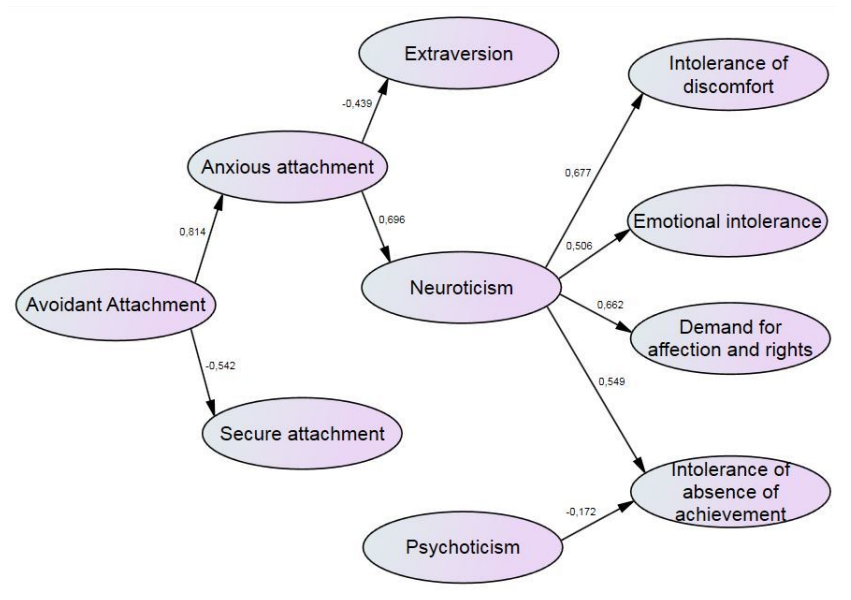

Figure 1. First Full Latent Variable Model

Figure 1 represents the first model with the standardized regression values $(\beta)$. It is observed that, in addition to the dimensions related to FI, extraversion and secure attachment resulted as dependent variables.

To analyze all direct and indirect effects, we calculated the matrix of total effects that can be observed in Table 5 . As variables with the most significant effects on the four dimensions of FI, neuroticism, anxious attachment, and avoidant attachment are highlighted. In addition, many explained variances were characterized by a large effect size as indicated by Cohen. 57

\section{MEASUREMENT INVARIANCE}

We performed multiple group analysis. In M2, factor loadings were constrained to be equal across groups; in M3, factor loadings and intercepts and, in M4, factor loadings, intercepts, and residuals. To evaluate measurement invariance, we used the changes of CFI and RMSEA. According to the content of Table 6 , it can be stated that all the changes 
Table 2. Regression results using FI as criteria and personality traits and attachment styles as predictors.

\begin{tabular}{|c|c|c|c|c|c|c|c|c|}
\hline \multirow[b]{2}{*}{ Criterion } & \multirow[b]{2}{*}{ Predictors } & \multirow[b]{2}{*}{$b$} & \multicolumn{2}{|c|}{$95 \% \mathrm{Cl}$} & \multirow[b]{2}{*}{ SE B } & \multirow[b]{2}{*}{$z$} & \multirow[b]{2}{*}{ Beta $(\beta)$} & \multirow[b]{2}{*}{$\mathrm{p}$} \\
\hline & & & LL & UL & & & & \\
\hline \multicolumn{9}{|c|}{ Emotional Intolerance } \\
\hline & Neuroticism & 0,951 & 0,365 & 1,538 & 0,299 & 3,177 & 0,336 & $0,001^{* *}$ \\
\hline & Extraversion & 0,075 & $-0,720$ & 0,870 & 0,406 & 0,185 & 0,017 & $0,853^{* *}$ \\
\hline & Psychoticism & $-0,077$ & $-0,481$ & 0,327 & 0,206 & $-0,373$ & $-0,033$ & $0,709^{* *}$ \\
\hline & Anxious attachment & 0,525 & 0,118 & 0,932 & 0,208 & 2,527 & 0,312 & $0,012^{* *}$ \\
\hline & Avoidant attachment & $-0,095$ & $-0,633$ & 0,444 & 0,275 & $-0,345$ & $-0,046$ & $0,730^{* *}$ \\
\hline & Secure attachment & 0,138 & $-0,165$ & 0,440 & 0,154 & 0,892 & 0,097 & $0,373^{* *}$ \\
\hline \multicolumn{9}{|c|}{ Demand for affection and rights } \\
\hline & Neuroticism & 0,909 & 0,291 & 1,526 & 0,315 & 2,883 & 0,298 & $0,004^{* *}$ \\
\hline & Extraversion & 0,540 & $-0,346$ & 1,426 & 0,452 & 1,195 & 0,114 & $0,232^{* *}$ \\
\hline & Psychoticism & 0,160 & $-0,259$ & 0,579 & 0,214 & 0,748 & 0,064 & $0,454^{* *}$ \\
\hline & Anxious attachment & 0,360 & $-0,107$ & 0,826 & 0,238 & 1,510 & 0,199 & $0,131^{* *}$ \\
\hline & Avoidant attachment & 0,190 & $-0,483$ & 0,862 & 0,343 & 0,553 & 0,085 & $0,580^{* *}$ \\
\hline & Secure attachment & $-0,344$ & $-0,721$ & 0,032 & 0,192 & $-1,793$ & $-0,226$ & $0,073^{* *}$ \\
\hline \multicolumn{9}{|c|}{ Intolerance of discomfort } \\
\hline & Neuroticism & 0,445 & 0,075 & 0,815 & 0,189 & 2,360 & 0,274 & $0,018^{* *}$ \\
\hline & Extraversion & 0,170 & $-0,326$ & 0,666 & 0,253 & 0,672 & 0,067 & $0,501^{* *}$ \\
\hline & Psychoticism & $-0,175$ & $-0,411$ & 0,061 & 0,120 & $-1,451$ & $-0,130$ & $0,147^{* *}$ \\
\hline & Anxious attachment & 0,377 & 0,050 & 0,704 & 0,167 & 2,258 & 0,391 & $0,024^{* *}$ \\
\hline & Avoidant attachment & 0,145 & $-0,248$ & 0,539 & 0,201 & 0,724 & 0,122 & $0,469^{* *}$ \\
\hline & Secure attachment & 0,067 & $-0,096$ & 0,229 & 0,083 & 0,805 & 0,082 & $0,421^{* *}$ \\
\hline \multicolumn{9}{|c|}{ Intolerance to absence of achievement } \\
\hline & Neuroticism & 0,826 & 0,186 & 1,465 & 0,326 & 2,531 & 0,277 & $0,011^{* *}$ \\
\hline & Extraversion & 0,667 & $-0,278$ & 1,612 & 0,482 & 1,384 & 0,144 & $0,166^{* *}$ \\
\hline & Psychoticism & $-0,502$ & $-0,984$ & $-0,020$ & 0,246 & $-2,042$ & $-0,204$ & $0,041^{* *}$ \\
\hline & Anxious attachment & 0,333 & $-0,162$ & 0,828 & 0,253 & 1,318 & 0,188 & $0,188^{* *}$ \\
\hline & Avoidant attachment & 0,306 & $-0,391$ & 1,003 & 0,355 & 0,861 & 0,140 & $0,389^{* *}$ \\
\hline & Secure attachment & $-0,097$ & $-0,439$ & 0,246 & 0,175 & $-0,554$ & $-0,065$ & $0,580^{* *}$ \\
\hline
\end{tabular}

Notes. $\mathrm{n}=255 ;{ }^{* * *} \mathrm{p}<.01 ; * \mathrm{p}<.05$; CI, confidence interval; LI, lower limit; UL, upper limit.

were within acceptable ranges with $|\Delta \mathrm{CFI}| \leqslant 0.010$ according to Cheung and Rensvold, 58 and $\mid \triangle$ RMSEA $\mid \leqslant 0.015$ according to Chen, ${ }^{59}$ so we can affirm that the model evaluates, regardless of gender, the same construct.

\section{DEVELOPMENT OF THE SECOND STRUCTURAL MODEL}

Following the second objective, an alternative model was developed to assess whether secure attachment can decrease the likelihood of maladaptive attachment styles arising and, consequently, FI arising. We used the Backwards Elimination technique since this variable selection procedure begins with the global model that does not assume bias. ${ }^{60}$ As a stopping rule, the inclusion of secure attachment was established.

According to the results of Table 2, we eliminated the variables without direct effects on the dimensions of FI and calculated the regression values again. We noted that the direct effect of psychoticism on discomfort intolerance and the direct effect of anxious attachment on emotional intolerance were no longer significant and therefore eliminated them.

To include extraversion, avoidant attachment, and secure attachment in the model, we added these factors as independent variables and anxious attachment, neuroticism, and psychoticism as dependent variables. As can be seen in Table $\underline{7}$, there were several probability values $\geqslant 0.05$, so we eliminated the respective effects of the model: Extraversion on neuroticism, secure attachment on neuroticism, secure attachment on anxious attachment, extraversion on psychoticism, and secure attachment on psychoticism. After these modifications, avoidance attachment no longer had a significant effect on psychoticism, so it was excluded from the model.

\section{ESTABLISHMENT OF THE SECOND STRUCTURAL MODEL.}

To include the secure attachment in the model, we analyzed whether this factor directly affected extraversion and 
Table 3. Regression results using personality traits and attachment styles as criteria and predictors

\begin{tabular}{|c|c|c|c|c|c|c|c|c|}
\hline \multirow[b]{2}{*}{ Criterion } & \multirow[b]{2}{*}{ Predictors } & \multirow[b]{2}{*}{$b$} & \multicolumn{2}{|c|}{$95 \% \mathrm{Cl}$} & \multirow[b]{2}{*}{ SE B } & \multirow[b]{2}{*}{$z$} & \multirow[b]{2}{*}{$\operatorname{Beta}(\beta)$} & \multirow[b]{2}{*}{$\mathrm{p}$} \\
\hline & & & $\mathrm{LL}$ & UL & & & & \\
\hline \multicolumn{9}{|c|}{ Neuroticism } \\
\hline & Extraversion & $-0,151$ & $-0,551$ & 0,249 & 0,204 & $-0,740$ & $-0,091$ & $0,459^{* *}$ \\
\hline & Psychoticism & 0,020 & $-0,120$ & 0,159 & 0,071 & 0,275 & 0,026 & $0,784^{* *}$ \\
\hline & Anxious attachment & 0,054 & $-0,139$ & 0,247 & 0,098 & 0,549 & 0,091 & $0,583^{* *}$ \\
\hline & Avoidant attachment & 0,185 & $-0,054$ & 0,423 & 0,122 & 1,517 & 0,278 & $0,129^{* *}$ \\
\hline & Secure attachment & $-0,017$ & $-0,142$ & 0,109 & 0,064 & $-0,259$ & $-0,033$ & $0,796^{* *}$ \\
\hline \multicolumn{9}{|c|}{ Extraversion } \\
\hline & Neuroticism & $-0,051$ & $-0,186$ & 0,083 & 0,069 & $-0,749$ & $-0,086$ & $0,454^{* *}$ \\
\hline & Psychoticism & 0,035 & $-0,058$ & 0,128 & 0,047 & 0,738 & 0,077 & $0,460^{* *}$ \\
\hline & Anxious attachment & $-0,151$ & $-0,277$ & $-0,026$ & 0,064 & $-2,357$ & $-0,428$ & $0,018^{* *}$ \\
\hline & Avoidant attachment & $-0,024$ & $-0,159$ & 0,111 & 0,069 & $-0,347$ & $-0,060$ & $0,729^{* *}$ \\
\hline & Secure attachment & $-0,033$ & $-0,123$ & 0,058 & 0,046 & $-0,706$ & $-0,107$ & $0,480^{* *}$ \\
\hline \multicolumn{9}{|c|}{ Psychoticism } \\
\hline & Neuroticism & 0,038 & $-0,228$ & 0,304 & 0,136 & 0,277 & 0,028 & $0,782^{* *}$ \\
\hline & Extraversion & 0,198 & $-0,339$ & 0,734 & 0,274 & 0,723 & 0,089 & $0,470^{* *}$ \\
\hline & Anxious attachment & $-0,027$ & $-0,248$ & 0,193 & 0,113 & $-0,242$ & $-0,035$ & $0,809^{* *}$ \\
\hline & Avoidant attachment & 0,309 & 0,055 & 0,563 & 0,130 & 2,382 & 0,350 & $0,017^{* *}$ \\
\hline & Secure attachment & 0,187 & $-0,014$ & 0,388 & 0,102 & 1,821 & 0,278 & $0,069^{* *}$ \\
\hline \multicolumn{9}{|c|}{ Anxious attachment } \\
\hline & Neuroticism & 0,083 & 0,152 & $-0,214$ & 0,380 & 0,547 & 0,049 & $0,584^{* *}$ \\
\hline & Extraversion & $-0,685$ & 0,294 & $-1,262$ & $-0,109$ & $-2,330$ & $-0,243$ & $0,020^{* *}$ \\
\hline & Psychoticism & $-0,022$ & 0,090 & $-0,198$ & 0,155 & $-0,242$ & $-0,017$ & $0,809^{* *}$ \\
\hline & Avoidant attachment & 0,602 & 0,157 & 0,294 & 0,910 & 3,829 & 0,535 & $0,000^{* *}$ \\
\hline & Secure attachment & $-0,111$ & 0,088 & $-0,284$ & 0,063 & $-1,251$ & $-0,129$ & $0,211^{* *}$ \\
\hline \multicolumn{9}{|c|}{ Avoidant attachment } \\
\hline & Neuroticism & 0,210 & $-0,063$ & 0,483 & 0,139 & 1,510 & 0,140 & $0,131^{* *}$ \\
\hline & Extraversion & $-0,080$ & $-0,540$ & 0,380 & 0,235 & $-0,341$ & $-0,032$ & $0,733^{* *}$ \\
\hline & Psychoticism & 0,183 & 0,006 & 0,360 & 0,090 & 2,024 & 0,161 & $0,043^{* *}$ \\
\hline & Anxious attachment & 0,446 & 0,194 & 0,699 & 0,129 & 3,465 & 0,502 & $0,001^{* *}$ \\
\hline & Secure attachment & $-0,207$ & $-0,388$ & $-0,027$ & 0,092 & $-2,252$ & $-0,272$ & $0,024^{* *}$ \\
\hline \multicolumn{9}{|c|}{ Secure attachment } \\
\hline & Neuroticism & $-0,052$ & $-0,447$ & 0,343 & 0,202 & $-0,258$ & $-0,026$ & $0,796^{* *}$ \\
\hline & Extraversion & $-0,301$ & $-1,134$ & 0,532 & 0,425 & $-0,708$ & $-0,091$ & $0,479^{* *}$ \\
\hline & Psychoticism & 0,305 & 0,019 & 0,592 & 0,146 & 2,091 & 0,205 & $0,037^{* *}$ \\
\hline & Anxious attachment & $-0,226$ & $-0,558$ & 0,105 & 0,169 & $-1,337$ & $-0,194$ & $0,181^{* *}$ \\
\hline & Avoidant attachment & $-0,572$ & $-0,969$ & $-0,175$ & 0,202 & $-2,826$ & $-0,436$ & $0,005^{* *}$ \\
\hline
\end{tabular}

Notes. $\mathrm{n}=255 ; * * \mathrm{p}<.01 ; * \mathrm{p}<.05$; CI, Confidence interval; LI, lower limit; UL, upper limit.

avoidant attachment. Table 8 represents the regression values of the final model. The following direct effects exhibited the highest standardized values $(\beta)$ : secure attachment on avoidant attachment, avoidant attachment on anxious attachment, and neuroticism on demand for affection and rights.

Next, we evaluated the fit indices and found that the second model obtained favorable indices according to the indications of $\mathrm{Hu}$ and Bentler: ${ }^{50} \chi 2 \mathrm{WLSMV}=448.859$; $\mathrm{df}=387$; Scaling $=1.357 ; \mathrm{p}=0.016 ;$ RMSEA $=0.025,90 \%$ CI $[0.012$; $0.035] ; \mathrm{SRMR}=0.055 ; \mathrm{CFI}=0.956$ and TLI $=0.950$. Figure 2 represents the second model with the respective standardized regression values.

To deepen the analysis of the model developed, the matrix of total effects was calculated, which comprises the direct and indirect effects. Table 9 shows that neuroticism had the most considerable effects on the four dimensions of FI. The most significant effects that neuroticism received were the positive effect of avoidant attachment and the negative impact of secure attachment. On the other hand, there was a significant effect that anxious attachment had on intolerance 
Table 4. Regression results of the Full Latent Variable Model

\begin{tabular}{|c|c|c|c|c|c|c|c|c|}
\hline \multirow[b]{2}{*}{ Criterion } & \multirow[b]{2}{*}{ Predictors } & \multirow[b]{2}{*}{$b$} & \multicolumn{2}{|c|}{$95 \% \mathrm{Cl}$} & \multirow[b]{2}{*}{ SE B } & \multirow[b]{2}{*}{$z$} & \multirow[b]{2}{*}{$\begin{array}{l}\text { Beta } \\
(\beta)\end{array}$} & \multirow[b]{2}{*}{$\mathrm{p}$} \\
\hline & & & LL & UL & & & & \\
\hline \multicolumn{9}{|c|}{ Intolerance of discomfort } \\
\hline & Neuroticism & 1,546 & 0,804 & 2,288 & 0,379 & 4,083 & 0,677 & $0,000^{* *}$ \\
\hline \multicolumn{9}{|c|}{ Emotional intolerance } \\
\hline & Neuroticism & 1,997 & 1,119 & 2,874 & 0,448 & 4,460 & 0,506 & $0,000^{* *}$ \\
\hline \multicolumn{9}{|c|}{ Demand for affection and rights } \\
\hline & Neuroticism & 2,807 & 1,687 & 3,928 & 0,572 & 4,911 & 0,662 & $0,000^{* *}$ \\
\hline \multicolumn{9}{|c|}{ Intolerance to absence of achievement } \\
\hline & Neuroticism & 2,285 & 1,278 & 3,291 & 0,514 & 4,448 & 0,549 & $0,000^{* *}$ \\
\hline & Psychoticism & $-0,388$ & $-0,750$ & $-0,026$ & 0,185 & $-2,102$ & $-0,172$ & $0,036^{* *}$ \\
\hline \multicolumn{9}{|c|}{ Neuroticism } \\
\hline & $\begin{array}{l}\text { Anxious } \\
\text { attachment }\end{array}$ & 0,321 & 0,189 & 0,453 & 0,067 & 4,767 & 0,696 & $0,000^{* *}$ \\
\hline \multicolumn{9}{|c|}{ Extraversion } \\
\hline & $\begin{array}{l}\text { Anxious } \\
\text { attachment }\end{array}$ & $-0,179$ & $-0,282$ & $-0,076$ & 0,052 & $-3,416$ & $-0,439$ & $0,001^{* *}$ \\
\hline \multicolumn{9}{|c|}{ Anxious attachment } \\
\hline & $\begin{array}{l}\text { Avoidant } \\
\text { attachment }\end{array}$ & 0,925 & 0,542 & 1,308 & 0,195 & 4,737 & 0,814 & $0,000^{* *}$ \\
\hline \multicolumn{9}{|c|}{ Secure attachment } \\
\hline & $\begin{array}{l}\text { Avoidant } \\
\text { attachment }\end{array}$ & $-0,798$ & $-1,214$ & $-0,383$ & 0,212 & $-3,766$ & $-0,542$ & $0,000^{* *}$ \\
\hline
\end{tabular}

Notes. $\mathrm{n}=255 ; * * \mathrm{p}<.01 ; * \mathrm{p}<.05$; CI, Confidence interval; LI, lower limit; UL, upper limit.

Table 5. Matrix of standardized total effects of the first Full Latent Variable Model

\begin{tabular}{|c|c|c|c|c|c|}
\hline \multirow[b]{2}{*}{ Criterions } & \multicolumn{4}{|c|}{ Predictors } & \multirow[b]{2}{*}{$\mathrm{R}^{2}$} \\
\hline & $\begin{array}{l}\text { Avoidant } \\
\text { attachment }\end{array}$ & $\begin{array}{l}\text { Anxious } \\
\text { attachment }\end{array}$ & Psychoticism & Neuroticism & \\
\hline (1) Anxious attachment & 0,814 & 0,000 & 0,000 & 0,000 & 0,663 \\
\hline (2) Neuroticism & 0,567 & 0,696 & 0,000 & 0,000 & 0,484 \\
\hline (3) Secure attachment & $-0,542$ & 0,000 & 0,000 & 0,000 & 0,294 \\
\hline (4) Extraversion & $-0,357$ & $-0,439$ & 0,000 & 0,000 & 0,192 \\
\hline $\begin{array}{l}\text { (5) Intolerance to absence of } \\
\text { achievement }\end{array}$ & 0,311 & 0,382 & $-0,172$ & 0,549 & 0,322 \\
\hline $\begin{array}{l}\text { (6) Demand for affection and } \\
\text { rights }\end{array}$ & 0,375 & 0,461 & 0,000 & 0,662 & 0,438 \\
\hline (7) Emotional intolerance & 0,287 & 0,352 & 0,000 & 0,506 & 0,256 \\
\hline (8) Intolerance of discomfort & 0,384 & 0,471 & 0,000 & 0,677 & 0,458 \\
\hline FI mean (5-8) & 0,339 & 0,417 & $-0,043$ & 0,599 & - \\
\hline
\end{tabular}

Notes. $\mathrm{n}=255$

to discomfort.

\section{MEASUREMENT INVARIANCE}

According to the content of Table 10 , it can be stated that the second model represents measurement invariance according to gender since the changes in the fit indices were found within adequate ranges with $|\triangle \mathrm{CFI}| \leqslant 0.010$ according to Cheung and Rensvold, 58 and $\mid \triangle$ RMSEA $\mid \leqslant 0.015$ according to Chen. 59 
Table 6. Model fit and model comparisons testing for measurement invariance of the first Full Latent Variable Model regarding gender

\begin{tabular}{|c|c|c|c|c|c|c|c|c|c|c|c|}
\hline \multirow[t]{2}{*}{ Model } & \multicolumn{6}{|l|}{ Model fit } & \multicolumn{5}{|c|}{ Model comparisons } \\
\hline & $x 2^{\text {WLSMV }}$ & $\mathrm{df}$ & $x^{2 / d f}$ & Scaling & RMSEA & $\mathrm{CFI}$ & $\Delta \times 2^{\text {WLSMV }}$ & $\Delta \mathrm{df}$ & $\mathrm{p}$ & $\triangle \mathrm{RMSEA}$ & $\Delta \mathrm{CFI}$ \\
\hline M1: Configural & 788,084 & 760 & 1,037 & 1,672 & 0,017 & 0,976 & & & & & \\
\hline M2: Weak & 805,938 & 780 & 1,033 & 1,908 & 0,016 & 0,978 & 17,854 & 20 & 0,597 & $-0,001$ & 0,002 \\
\hline M3: Strong & 831,309 & 800 & 1,039 & 1,914 & 0,018 & 0,973 & 25,371 & 20 & 0,188 & 0,002 & $-0,005$ \\
\hline M4: Strict & 863,607 & 830 & 1,040 & 1,964 & 0,018 & 0,971 & 32,298 & 30 & 0,354 & 0,000 & $-0,002$ \\
\hline
\end{tabular}

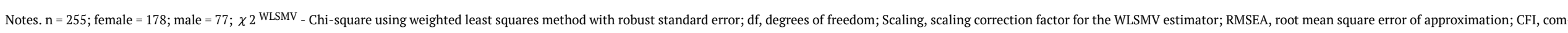
parative fit index. 
Table 7. Regression results using FI as criteria and personality and attachment styles as predictors, including possible indirect effects.

\begin{tabular}{|c|c|c|c|c|c|c|c|c|}
\hline \multirow[b]{2}{*}{ Criterion } & \multirow[b]{2}{*}{ Predictors } & \multirow[b]{2}{*}{$b$} & \multicolumn{2}{|c|}{$95 \% \mathrm{Cl}$} & \multirow[b]{2}{*}{ SE B } & \multirow[b]{2}{*}{ z } & \multirow[b]{2}{*}{ Beta $(\beta)$} & \multirow[b]{2}{*}{$\mathrm{p}$} \\
\hline & & & LL & UL & & & & \\
\hline \multicolumn{9}{|c|}{ Intolerance of discomfort } \\
\hline & Neuroticism & 0,837 & 0,220 & 1,455 & 0,315 & 2,657 & 0,386 & $0,008^{* *}$ \\
\hline & Anxious attachment & 0,243 & 0,010 & 0,476 & 0,119 & 2,046 & 0,254 & $0,041^{* *}$ \\
\hline \multicolumn{9}{|c|}{ Emotional intolerance } \\
\hline & Neuroticism & 1,835 & 1,002 & 2,667 & 0,425 & 4,317 & 0,485 & $0,000^{* *}$ \\
\hline \multicolumn{9}{|c|}{ Intolerance to absence of achievement } \\
\hline & Psychoticism & $-0,456$ & $-0,804$ & $-0,109$ & 0,177 & $-2,577$ & $-0,191$ & $0,010^{* *}$ \\
\hline & Neuroticism & 2,237 & 1,242 & 3,231 & 0,507 & 4,408 & 0,564 & $0,000^{* *}$ \\
\hline \multicolumn{9}{|c|}{ Demand for affection and rights } \\
\hline & Neuroticism & 2,834 & 1,735 & 3,934 & 0,561 & 5,053 & 0,697 & $0,000^{* *}$ \\
\hline \multicolumn{9}{|c|}{ Anxious attachment } \\
\hline & Extraversion & $-0,617$ & $-1,166$ & $-0,069$ & 0,280 & $-2,206$ & $-0,226$ & $0,027^{* *}$ \\
\hline & Avoidant attachment & 0,882 & 0,411 & 1,353 & 0,240 & 3,671 & 0,667 & $0,000^{* *}$ \\
\hline & Secure attachment & $-0,079$ & $-0,271$ & 0,114 & 0,098 & $-0,801$ & $-0,092$ & $0,423^{* *}$ \\
\hline \multicolumn{9}{|c|}{ Neuroticism } \\
\hline & Extraversion & $-0,032$ & $-0,269$ & 0,205 & 0,121 & $-0,266$ & $-0,027$ & $0,790^{* *}$ \\
\hline & Avoidant attachment & 0,342 & 0,130 & 0,555 & 0,108 & 3,163 & 0,588 & $0,002^{* *}$ \\
\hline & Secure attachment & $-0,037$ & $-0,129$ & 0,055 & 0,047 & $-0,785$ & $-0,098$ & $0,433^{* *}$ \\
\hline \multicolumn{9}{|c|}{ Psychoticism } \\
\hline & Extraversion & 0,169 & $-0,266$ & 0,604 & 0,222 & 0,762 & 0,085 & $0,446^{* *}$ \\
\hline & Avoidant attachment & 0,304 & 0,013 & 0,595 & 0,148 & 2,047 & 0,314 & $0,041^{* *}$ \\
\hline & Secure attachment & 0,181 & $-0,017$ & 0,379 & 0,101 & 1,795 & 0,291 & $0,073^{* *}$ \\
\hline
\end{tabular}

Notes. $\mathrm{n}=255$; *** $\mathrm{p}<.01 ; * \mathrm{p}<.05$; CI, Confidence interval; LL, lower limit; UL, upper limit.

\section{DISCUSSION}

To determine the relationships between attachment styles, personality traits, and FI, this research developed two accurate models that determine the most significant FI predictors. In addition, the factorial invariance of both models has been verified so that their results represent the same construct, independent of gender.

The first model was developed to maximize the explained variances; priority was given to the significance level of the effects ( $\mathrm{p}$ and $\beta$ ). Neuroticism, anxious attachment, and avoidant attachment were found to be the strongest predictors of FI. Even so, three essential aspects must be taken into account: (1) only neuroticism had a direct effect on FI, (2) only anxious attachment had a direct effect on neuroticism, and (3) avoidant attachment was the only independent variable of the model.

Regarding point (1), previous studies found a positive association between both variables, ${ }^{16-18}$ but the present investigation could account for its direct association, showing a high probability that neuroticism is a link between FI and the other variables. Since neuroticism exhibits correlations with FI and many different conditions six, it is necessary to determine which factors favor increased levels of this personality trait. This need leads directly to the analysis of

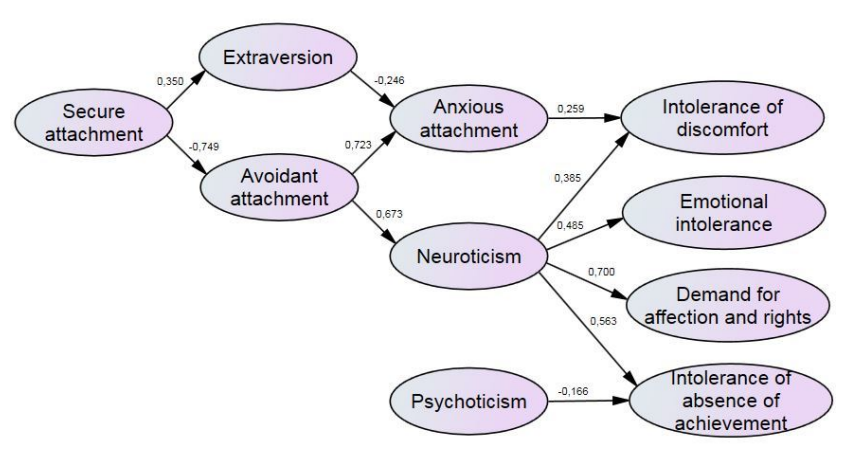

Figure 2. Second Full Latent Variable Model

points (2) and (3). Noftle and Shaver and Jenkins-Guarnieri, and colleagues found significant associations between $a n x-$ ious attachment and neuroticism, 31,32 so the present study results are in line with previous findings. Nonetheless, we should bear in mind that Jenkins-Guarnieri and colleagues found no correlations between avoidant attachment and neuroticism and, ${ }^{32}$ according to Noftle and Shaver's summary, the associations found between these two factors were either weak or non-existent. ${ }^{31}$

Following the first model developed, an explanation can 
Table 8. Regression results of the second Full Latent Variable Model

\begin{tabular}{|c|c|c|c|c|c|c|c|c|}
\hline \multirow[b]{2}{*}{ Criterion } & \multirow[b]{2}{*}{ Predictors } & \multirow[b]{2}{*}{$b$} & \multicolumn{2}{|c|}{$95 \% \mathrm{Cl}$} & \multirow[b]{2}{*}{ SE B } & \multirow[b]{2}{*}{ z } & \multirow[b]{2}{*}{$\begin{array}{c}\text { Beta } \\
(\beta)\end{array}$} & \multirow[b]{2}{*}{$\mathrm{p}$} \\
\hline & & & LL & UL & & & & \\
\hline \multicolumn{9}{|c|}{ Intolerance of discomfort } \\
\hline & Neuroticism & 0,837 & 0,217 & 1,456 & 0,316 & 2,646 & 0,385 & $0,008^{* *}$ \\
\hline & $\begin{array}{l}\text { Anxious } \\
\text { attachment }\end{array}$ & 0,247 & 0,010 & 0,485 & 0,121 & 2,039 & 0,259 & $0,041^{* *}$ \\
\hline \multicolumn{9}{|c|}{ Emotional intolerance } \\
\hline & Neuroticism & 1,841 & 1,009 & 2,672 & 0,424 & 4,338 & 0,485 & $0,000^{* *}$ \\
\hline \multicolumn{9}{|c|}{ Demand for affection and rights } \\
\hline & Neuroticism & 2,853 & 1,757 & 3,948 & 0,559 & 5,105 & 0,700 & $0,000^{* *}$ \\
\hline \multicolumn{9}{|c|}{ Intolerance to absence of achievement } \\
\hline & Psychoticism & $-0,386$ & $-0,758$ & $-0,013$ & 0,190 & $-2,030$ & $-0,166$ & $0,042^{* *}$ \\
\hline & Neuroticism & 2,240 & 1,252 & 3,228 & 0,504 & 4,444 & 0,563 & $0,000^{* *}$ \\
\hline \multicolumn{9}{|c|}{ Anxious attachment } \\
\hline & Extraversion & $-0,658$ & $-1,153$ & $-0,163$ & 0,252 & $-2,608$ & $-0,246$ & $0,009^{* *}$ \\
\hline & $\begin{array}{l}\text { Avoidant } \\
\text { attachment }\end{array}$ & 0,988 & 0,525 & 1,451 & 0,236 & 4,182 & 0,723 & $0,000^{* *}$ \\
\hline \multicolumn{9}{|c|}{ Neuroticism } \\
\hline & $\begin{array}{l}\text { Avoidant } \\
\text { attachment }\end{array}$ & 0,404 & 0,211 & 0,597 & 0,099 & 4,103 & 0,673 & $0,000^{* *}$ \\
\hline \multicolumn{9}{|c|}{ Extraversion } \\
\hline & Secure attachment & 0,136 & 0,023 & 0,249 & 0,058 & 2,351 & 0,350 & $0,019^{* *}$ \\
\hline \multicolumn{9}{|c|}{ Avoidant attachment } \\
\hline & Secure attachment & $-0,569$ & $-0,933$ & $-0,205$ & 0,186 & $-3,067$ & $-0,749$ & $0,002^{* *}$ \\
\hline
\end{tabular}

Notes. $\mathrm{n}=255$; ${ }^{* * *} \mathrm{p}<.01 ;{ }^{*} \mathrm{p}<.05$; CI, Confidence interval; LL, lower limit; UL, upper limit.

be given to the weak association between avoidant attachment and neuroticism since its effect is not direct. According to this model, which maximizes the explained variances, avoidance attachment is the only independent variable, so this factor predicts high levels of anxious attachment, through this, high degrees of neuroticism and FI. This last result becomes even more important when considering that avoidant and anxious attachment explains $48.4 \%$ of the variance of neuroticism. Likewise, $45.8 \%$ of the variance corresponding to intolerance to discomfort and $43.8 \%$ of the variance of demand for affection and rights are explained through these three factors. These percentages could be considered high since a psychometric instrument must explain $60 \%,{ }^{55}$ or $50 \%$ of its variance. ${ }^{61}$

We developed the second model interpreting secure attachment as a protective element, and, ${ }^{36}$ to use this factor as the only independent variable, the Backwards Elimination method was used. ${ }^{60}$ Like the first model, neuroticism and avoidant attachment played primary roles. Even so, there were three differences. Firstly, the protective factor of secure attachment is in line with previous studies, ${ }^{36}$ and Bowlby's attachment theory; ${ }^{62}$ furthermore, the diminished importance of anxious attachment in predicting the four dimensions of FI and, essentially, the relationship between the two maladaptive attachment styles. Although high levels of avoidant attachment continue to predict high anxious attachment significantly, the latter receives a moderating effect from extraversion. That shows that an individual with low levels of extraversion will be more prone to developing anxious attachment. While numerous studies found correlations between extraversion and these two attachment styles, ${ }^{31,63}$ this research described the relationship between them in more detail. Likewise, most of the effects of anxious attachment appear to be on intolerance of discomfort. 
Table 9. Matrix of standardized total effects of the second Full Latent Variable Model

\begin{tabular}{|c|c|c|c|c|c|c|c|}
\hline \multirow[b]{2}{*}{ Criterions } & \multicolumn{6}{|c|}{ Predictors } & \multirow[b]{2}{*}{$\mathrm{R}^{2}$} \\
\hline & Secure attachment & Avoidant attachment & Extraversion & Psychoticism & Anxious attachment & Neuroticism & \\
\hline (1) Avoidant attachment & $-0,749$ & 0,000 & 0,000 & 0,000 & 0,000 & 0,000 & 0,562 \\
\hline (2) Extraversion & 0,350 & 0,000 & 0,000 & 0,000 & 0,000 & 0,000 & 0,122 \\
\hline (3) Anxious attachment & $-0,628$ & 0,723 & $-0,246$ & 0,000 & 0,000 & 0,000 & 0,677 \\
\hline (4) Neuroticism & $-0,504$ & 0,673 & 0,000 & 0,000 & 0,000 & 0,000 & 0,452 \\
\hline (5) Intolerance of absence of achievement & $-0,284$ & 0,379 & 0,000 & $-0,166$ & 0,000 & 0,563 & 0,339 \\
\hline (6) Demand for affection and rights & $-0,353$ & 0,471 & 0,000 & 0,000 & 0,000 & 0,700 & 0,490 \\
\hline (7) Emotional intolerance & $-0,244$ & 0,326 & 0,000 & 0,000 & 0,000 & 0,485 & 0,235 \\
\hline (8) Intolerance of discomfort & $-0,357$ & 0,446 & $-0,064$ & 0,000 & 0,259 & 0,385 & 0,321 \\
\hline Mean of FI (5-8) & $-0,309$ & 0,406 & $-0,016$ & $-0,042$ & 0,065 & 0,533 & - \\
\hline
\end{tabular}

Notes. $\mathrm{n}=255$.

Table 10. Model fit and model comparisons testing for measurement invariance of the second Full Latent Variable Model regarding gender

\begin{tabular}{|c|c|c|c|c|c|c|c|c|c|c|c|}
\hline \multirow[t]{2}{*}{ Model } & \multicolumn{6}{|l|}{ Model fit } & \multicolumn{5}{|c|}{ Model comparison } \\
\hline & $x^{2}$ WLSMV & $\mathrm{df}$ & $x 2 / d f$ & Scaling & RMSEA & $\mathrm{CFI}$ & $\Delta \times 2^{W L S M V}$ & $\Delta \mathrm{df}$ & $\mathrm{p}$ & $\triangle \mathrm{RMSEA}$ & $\Delta \mathrm{CFI}$ \\
\hline M1: Configural & 823,264 & 774 & 1,064 & 1,730 & 0,022 & 0,958 & & & & & \\
\hline M2: Weak & 837,505 & 794 & 1,055 & 1,965 & 0,021 & 0,963 & 14,241 & 20 & 0,818 & $-0,001$ & 0,005 \\
\hline M3: Strong & 862,712 & 814 & 1,060 & 1,971 & 0,022 & 0,958 & 25,207 & 20 & 0,194 & 0,001 & $-0,005$ \\
\hline M4: Strict & 895,464 & 844 & 1,061 & 2,020 & 0,022 & 0,956 & 32,752 & 30 & 0,333 & 0,000 & $-0,002$ \\
\hline
\end{tabular}

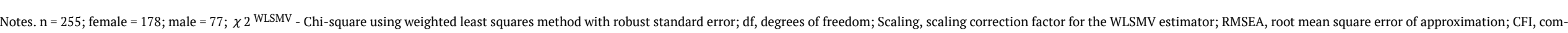
parative fit index. 
Let's consider the two models as a whole and, based on the high level of explained variances. We can affirm that maladaptive attachment styles and neuroticism play transcendent roles in predicting high levels of FI. The finding that attachment styles predict a very considerable proportion of the neuroticism variance requires the reinterpretation of this dimension. This statement is based on the origin of attachment styles since Bowlby affirmed that they develop during childhood and remain throughout life, ${ }^{64}$ although their intensity decreases. ${ }^{27}$ Interestingly, neuroticism and FI also tend to decline throughout life, 65 affirming the relationship between these constructs. Taking the points mentioned above as a whole, we can support that, to a great extent, high levels of neuroticism are due to an unfavorable attachment style, which has possibly developed during the individual's childhood. This conclusion highlights the importance of the interaction between the infant and the attachment figure since Ainsworth and colleagues describe that the attachment figures of children with secure attachment are characterized by a greater degree of sensitive attention. 30

If we compare the explained variance of extraversion with the explained variance of neuroticism, we can affirm that extraversion appears less related to attachment styles; its magnitude would not primarily depend on the interaction between the infant and the attachment figure.

It is noted that avoidant attachment occupies a transcendental position since its effect on secure attachment is more significant than the opposite effect. The same happens to the anxious attachment. Together with the relative independence of extraversion, this finding highlights that an avoidant behavior cannot be attributed to a personality trait but rather to a learned mechanism. The two models developed show that avoidant attachment initiates, in a certain way, a maladaptive process that leads to neuroticism and later to FI. In the strange situation, children with insecureavoidant attachment avoid, during reunification phases, the attachment figure's contact and instead focus their attention on the environment. Children with insecure-ambivalent attachment actively seek closeness with the attachment figure but, on the other hand, avoid the comforting behavior of their attachment figure, tend to get angry, and are difficult to calm down. ${ }^{29,30}$ As seen in these descriptions, children with insecure-ambivalent attachment also exhibit avoidant behavior, which is in line with the associations found in the present study.

Regarding psychoticism, we observed that it has a negative effect on intolerance to the absence of achievements, which means that, with high psychoticism, intolerance to the lack of achievements decreases. We can interpret that this effect was relatively mild compared to the positive impact that neuroticism had on this dimension of FI.

Regarding the limitations of this work, the sample size was relatively small, with non-probabilistic sampling conducted. Also, SEM does not show the existence of a causal relationship between certain factors, but instead, they can support a previous theory and/or must be validated with experimental designs. ${ }^{37,66}$ In this sense, the results of this research support the theories that indicate a complex interaction between the genetic components of behavior, such as personality (especially neuroticism and frustration) and environmental ones, in this case, the attachment styles. These factors can significantly attenuate or increase the genetic contributions of individual differences. ${ }^{26,67}$

\section{ACKNOWLEDGMENT}

*** Anonymous ***

\section{DATA ACCESSIBILITY STATEMENT}

The data that support the findings of this study are openly available at: https://osf.io/ ujqg3/?view_only=3429f93ace95455c9fb63e693fe15c7d

Submitted: May 31, 2021 EST, Accepted: June 03, 2021 EST 


\section{REFERENCES}

1. Amsel A. Frustration Theory. Cambridge University Press; 1992. doi:10.1017/CBO9780511665561

2. Mustaca AE. "Siento un dolor en el alma": ¿metáfora o realidad? Rev Argent Cienc Comport. 2013;5(2):47-60.

3. Ellis A. Rational-emotive therapy and cognitive behavior therapy: Similarities and differences. Cognit Ther Res. 1980;4(4):325-340. doi:10.1007/BF01178210

4. Tohver GC. Eysenck Giant Three. In: Carducci BJ, Nave CS, eds. The Wiley Encyclopedia of Personality and Individual Differences. 1st ed. John Wiley \& Sons Ltd.; 2020:155-159. doi:10.1002/9781119547174.ch20 $\underline{3}$

5. Eysenck HJ. Genetic and Environmental Contributions to Individual Differences: The Three Major Dimensions of Personality. J Pers. 1990;58(1):245-261. doi:10.1111/j.1467-6494.1990.tb 00915.X

6. Kotov R, Gamez W, Schmidt F, Watson D. Linking "big" personality traits to anxiety, depressive, and substance use disorders: A meta-analysis. Psychol Bull. 2010;136(5):768-821. doi:10.1037/a0020327

7. Latzman RD, Vaidya JG, Malikina VM, Berg JM, Lilienfeld SO. Exploring Associations Between Psychopathic Personality and Components of Disinhibition vs. Constraint. J Psychopathol Behav Assess. 2014;36(4):497-509. doi:10.1007/s10862-01 4-9415-4

8. Clark LA, Watson D, Mineka S. Temperament, personality, and the mood and anxiety disorders. $J$ Abnorm Psychol. 1994;103(1):103-116. doi:10.1037/00 21-843X.103.1.103

9. Khan AA, Jacobson KC, Gardner CO, Prescott CA, Kendler KS. Personality and comorbidity of common psychiatric disorders. Br J Psychiatry.

2005;186(3):190-196. doi:10.1192/bjp.186.3.190

10. Krueger RF, Markon KE. Reinterpreting Comorbidity: A Model-Based Approach to Understanding and Classifying Psychopathology. Annu Rev Clin Psychol. 2006;2(1):111-133. doi:10.114 6/annurev.clinpsy.2.022305.095213

11. Weinstock LM, Whisman MA. Neuroticism as a common feature of the depressive and anxiety disorders: A test of the revised integrative hierarchical model in a national sample. J Abnorm Psychol. 2006;115(1):68-74. doi:10.1037/0021-843X.1 15.1 .68
12. Liu F, Zhang Z, Chen L. Mediating effect of neuroticism and negative coping style in relation to childhood psychological maltreatment and smartphone addiction among college students in China. Child Abuse Negl. 2020;106:104531. doi:10.101 6/i.chiabu.2020.104531

13. Brickman AL, Yount SE, Blaney NT, Rothberg ST, De-Nour AK. Personality Traits and Long-Term Health. Psychosomatics. 1996;37(5):459-468. doi:10.1 016/S0033-3182(96)71534-7

14. Smith TW, MacKenzie J. Personality and risk of physical illness. Annu Rev Clin Psychol. 2006;2:435-467.

15. Spijker J, de Graaf R, Oldehinkel AJ, Nolen WA, Ormel J. Are the vulnerability effects of personality and psychosocial functioning on depression accounted for by subthreshold symptoms? Depress Anxiety. 2007;24(7):472-478. doi:10.1002/da.20252

16. Alba L, Giaquinto LG, Mustaca A. Intolerancia a la frustración y personalidad. Rev Argentina Ciencias del Comport. 2020;12(1):29-73.

17. Rivero LEM, Torrúbia R, Molina AJI, Torres C. Relationship between Frustration Intolerance and Personality Dimensions. Int J Psychol Psychol Ther. 2020;20(3):343-351.

18. Zajenkowska A, Zajenkowski M, Jankowski KS. The relationship between mood experienced during an exam, proneness to frustration and neuroticism. Learn Individ Differ. 2015;37(3):237-240. doi:10.1016/ j.lindif.2014.11.014

19. Cuenya L, Kamenetzky GV, Mustaca AE. Early experience and incentive relativity in adulthood. Int J Comp Psychol. 2014;27(3):459-473.

20. Flaherty C. Lack of Tolerance to ContrastReducing Actions of Chlordiazepoxide With Repeated Reward Reductions. Physiol Behav. 1996;60(2):653-656. doi:10.1016/0031-9384(96)0009 9-6

21. Fuentes-Verdugo E, Pellón R, Papini MR, Torres C, Fernández-Teruel A, Anselme P. Effects of partial reinforcement on autoshaping in inbred Roman highand low-avoidance rats. Physiol Behav.

2020;225:113111. doi:10.1016/i.physbeh.2020.113111

22. Serafini M, Ifran MC, Kamenetzky G, Cuenya L. Diferencias fenotípicas ante la devaluación y omisión de una recompensa esperada. Rev Psicol. 2020;29(2). $\underline{\mathrm{d}}$ oi:10.5354/0719-0581.2020.54743 
23. Papini S, R. Galatzer-Levy I, Papini MR. Identifying profiles of recovery from reward devaluation in rats. Behav Brain Res.

2014;275:212-218. doi:10.1016/j.bbr.2014.09.006

24. Xia W, Dymond S, Lloyd K, Vervliet B. Partial reinforcement of avoidance and resistance to extinction in humans. Behav Res Ther. 2017;96:79-89. doi:10.1016/j.brat.2017.04.002

25. Wesarg C, Van Den Akker AL, Oei NYL, Hoeve M, Wiers RW. Identifying pathways from early adversity to psychopathology: A review on dysregulated HPA axis functioning and impaired self-regulation in early childhood. Eur J Dev Psychol. 2020;17(6):808-827. do i:10.1080/17405629.2020.1748594

26. Maranges HM, Reynolds TA. Heritability. In: Carducci BJ, Nave CS, eds. The Wiley Encyclopedia of Personality and Individual Differences. Wiley; 2020:243-247. doi:10.1002/9781118970843.ch41

27. Bowlby J. Attachment and Loss, Vol. 1: Attachment. Basic books; 1969.

28. Schaffer HR, Emerson PE. The development of social attachments in infancy. Monogr Soc Res Child Dev. Published online 1964:1-77.

29. Ainsworth MD, Bell SM. Attachment, exploration, and separation: illustrated by the behavior of oneyear-olds in a strange situation. Child Dev. Published online 1970.

30. Ainsworth MD, Blehar M, Waters E, Wall S. Patterns of Attachment. Erlbaum; 1978.

31. Noftle EE, Shaver PR. Attachment dimensions and the big five personality traits: Associations and comparative ability to predict relationship quality. $J$ Res Pers. 2006;40(2):179-208. doi:10.1016/j.jrp.2004.1 $\underline{1.003}$

32. Jenkins-Guarnieri MA, Wright SL, Hudiburgh LM. The relationships among attachment style, personality traits, interpersonal competency, and Facebook use. J Appl Dev Psychol. 2012;33(6):294-301. doi:10.1016/j.appdev.2012.08.001

33. Barel E, Mizrachi Y, Nachmani M. Quantifying the Predictive Role of Temperament Dimensions and Attachment Styles on the Five Factor Model of Personality. Behav Sci (Basel). 2020;10(145):1-11. do i: $10.3390 / b s 10100145$

34. Mikulincer M, Shaver PR. The Attachment Behavioral System In Adulthood: Activation, Psychodynamics, And Interpersonal Processes. In: Zanna MP, ed. Advances in Experimental Social Psychology. 1st ed. Academic Press; 2003:53-152. do i:10.1016/S0065-2601(03)01002-5
35. Norberg MM, Crone C, Kwok C, Grisham JR. Anxious attachment and excessive acquisition: The mediating roles of anthropomorphism and distress intolerance. J Behav Addict. 2018;7(1):171-180. doi:1 $\underline{0.1556 / 2006.7 .2018 .08}$

36. Meyer B, Pilkonis PA, Proietti JM, Heape CL, Egan M. Attachment Styles and Personality Disorders as Predictors of Symptom Course. J Pers Disord. 2001;15(5):371-389. doi:10.1521/pedi.15.5.371.19200

37. Byrne BM. Structural Equation Modeling with AMOS. Routledge; 2016. doi:10.4324/9781315757421

38. Casullo MM, Liporace MF. Evaluación de los estilos de apego en adultos. Anu Investig. 2005;12:183-192.

39. Squillace M, Janeiro JP, Schmidt V. Adaptación local del Cuestionario Revisado de Personalidad de Eysenck (versión abreviada). Rev Evaluar. 2013;13(1):19-37.

40. Medrano LA, Franco P, Mustaca AE. Adaptación argentina de la escala "Escala de intolerancia a la frustración.” Behav Psychol Conduct. 2018;26(2):303-321.

41. Breunig MM, Kriegel HP, Ng RT, Sander J. LOF: Identifying density-based local outliers. ACM SIGMOD Rec. 2000;29(2):93-104. doi:10.1145/33519 $\underline{1.335388}$

42. Hahsler M, Piekenbrock M, Doran D. dbscan : Fast Density-Based Clustering with R. J Stat Softw. 2019;91(1):1-30. doi:10.18637/jss.v091.i01

43. Korkmaz S, Goksuluk D, Zararsiz G. MVN: An R Package for Assessing Multivariate Normality. $R J$. 2014;6(2):151-162. doi:10.32614/RJ-2014-031

44. Rosseel Y. lavaan: An R Package for Structural Equation Modeling. J Stat Softw. 2012;48(2):1-36. do i:10.18637/jss.v048.i02

45. Core Team. R: A Language and Environment for Statistical Computing.; 2020. https://www.r-project.or g/

46. Mardia VK. Measures of multivariate skewness and kurtosis with applications. Biometrika. 1970;57(3):519-530. doi:10.1093/biomet/57.3.519

47. Li CH. Confirmatory factor analysis with ordinal data: Comparing robust maximum likelihood and diagonally weighted least squares. Behav Res Methods. 2016;48(3):936-949. doi:10.3758/s13428-015-0619-7 
48. Bandalos DL. Relative Performance of Categorical Diagonally Weighted Least Squares and Robust Maximum Likelihood Estimation. Struct Equ Model A Multidiscip J. 2014;21(1):102-116. doi:10.1080/107055 11.2014.859510

49. Forero CG, Maydeu-Olivares A, Gallardo-Pujol D. Factor Analysis with Ordinal Indicators: A Monte Carlo Study Comparing DWLS and ULS Estimation. Struct Equ Model A Multidiscip J. 2009;16(4):625-641. doi:10.1080/10705510903203573

50. Hu L, Bentler PM. Cutoff criteria for fit indexes in covariance structure analysis: Conventional criteria versus new alternatives. Struct Equ Model A Multidiscip J. 1999;6(1):1-55. doi:10.1080/1070551990 $\underline{9540118}$

51. Preacher KJ, Hayes AF. Asymptotic and resampling strategies for assessing and comparing indirect effects in multiple mediator models. Behav Res Methods. 2008;40(3):879-891. doi:10.3758/BRM.4 $\underline{0.3 .879}$

52. Tofighi D, MacKinnon DP. Monte Carlo Confidence Intervals for Complex Functions of Indirect Effects. Struct Equ Model A Multidiscip J. 2016;23(2):194-205. doi:10.1080/10705511.2015.1057284

53. Fritz MS, Taylor AB, MacKinnon DP. Explanation of Two Anomalous Results in Statistical Mediation Analysis. Multivariate Behav Res. 2012;47(1):61-87. o $\underline{\mathrm{i}: 10.1080 / 00273171.2012 .640596}$

54. Creedon PS, Hayes AF. Small sample mediation analysis: How far can we push the bootstrap. In: Annual Conference of the Association for Psychological Science. ; 2015.

55. Hair JF, Black WC, Babin BJ, Anderson RE. Multivariate Data Analysis. 8th ed. Cengage Learning; 2019.

56. Saris WE, Satorra A, van der Veld WM. Testing Structural Equation Models or Detection of Misspecifications? Struct Equ Model A Multidiscip J. 2009;16(4):561-582. doi:10.1080/10705510903203433

57. Cohen J. Statistical Power Analysis for the Behavioral Sciences. 2nd ed. Lawrence Erlbaum Associates; 1988.
58. Cheung GW, Rensvold RB. Evaluating Goodnessof-Fit Indexes for Testing Measurement Invariance. Struct Equ Model A Multidiscip J. 2002;9(2):233-255. ㅁ oi:10.1207/S15328007SEM0902_5

59. Chen FF. Sensitivity of Goodness of Fit Indexes to Lack of Measurement Invariance. Struct Equ Model A Multidiscip J. 2007;14(3):464-504. doi:10.1080/107055 10701301834

60. Heinze G, Wallisch C, Dunkler D. Variable selection - A review and recommendations for the practicing statistician. Biometrical $J$. 2018;60(3):431-449. doi:10.1002/bimj.201700067

61. Merenda PF. A Guide to the Proper Use of Factor Analysis in the Conduct and Reporting of Research: Pitfalls to Avoid. Meas Eval Couns Dev. 1997;30(3):156-164. doi:10.1080/07481756.1997.1206 $\underline{8936}$

62. Bowlby J. Bindung: historische Wurzeln, theoretische Konzepte und klinische Relevanz. Die Bind Grundlagen, Forsch und Anwendung Klett-Cotta, Stuttgart. Published online 1995:17-29.

63. Gallo LC, Smith TW, Ruiz JM. An Interpersonal Analysis of Adult Attachment Style: Circumplex Descriptions, Recalled Developmental Experiences, Self-Representations, and Interpersonal Functioning in Adulthood. J Pers. 2003;71(2):141-182. doi:10.111 $\underline{1 / 1467-6494.7102003}$

64. Bowlby J. The nature of the child's tie to his mother. Int J Psychoanal. 1958;39:350-373.

65. Wortman J, Lucas RE, Donnellan MB. Stability and change in the Big Five personality domains: Evidence from a longitudinal study of Australians. Psychol Aging. 2012;27(4):867-874. doi:10.1037/a0029322

66. Kline RB. Principles and Practice of Structural Equation Modeling. 4th ed. The Guilford Press; 2015.

67. Barlow DH, Ellard KK, Sauer-Zavala S, Bullis JR, Carl JR. The Origins of Neuroticism. Perspect Psychol Sci. 2014;9(5):481-496. doi:10.1177/174569161454452 $\underline{8}$ 